\section{A general program for the calculation of the kappa coefficient}

\author{
MICHAEL J STRUBE \\ Washington University, St. Louis, Missouri
}

The kappa coefficient $(x)$ was developed by Cohen (1960) for the purpose of assessing chance-corrected interrater agreement on nominal data. In its simplest form, $x$ is defined as follows: $x=\left(p_{o}-p_{c}\right) /\left(1-p_{c}\right)$, where $p_{o}$ is the observed proportion of agreement and $p_{c}$ is the chance or expected proportion of agreement. Over the past three decades, $x$ has been extended to allow assessment among $K>2$ raters (e.g., Conger, 1980; Uebersax, 1982), to allow missing observations (e.g., Conger, 1980; Fleiss, 1971; Uebersax, 1982), to allow nonmutually exclusive response categories (i.e., subjects can be classified into more than one category; e.g., Fleiss, Spitzer, Endicott, \& Cohen, 1972; Kraemer, 1980), and to allow for partial disagreement (Cohen, 1968). The sampling distribution of $x$ has also been identified, allowing variance estimates to be calculated and significance tests to be conducted (Fleiss, Cohen, \& Everitt, 1969). Not surprisingly, several computer programs have been offered as efficient means of providing one or more of these $x$ features (e.g., Bloor, 1983; Burns \& Cavallaro, 1982; Chan, 1987; Powers, 1985; Watkins \& Larimer, 1980).

The present program provides a more comprehensive package by including features found in these earlier programs, while including additional features as well. The program provides for the calculation of $x$ and weighted $x$ for any number of raters (subject to the memory constraints of the machine being used). When the number of raters is greater than 2, all pairwise $\varkappa$ are also provided. Missing data in the matrix of observations are handled in the calculation of multiple-rater $x$ by using Uebersax's (1982) generalized approach. This approach is based on the calculation of weighted averages of pairwise estimates for observed agreement $\left(p_{o}\right)$ and chance agreement $\left(p_{c}\right)$, each weighted by the number of subjects each pair of judges has in common. For weighted $x$, the user supplies a weight matrix that indicates the degree of partial credit to be given for different types of disagreement. The careful construction of the weight matrix also allows the calculation of $x$ when subjects can be classified into more than one category. This arises, for example, in the case of medical or psychiatric diagnosis where more than one condition may be present in the same subject. Relative ordering of these multiple diagnoses is also allowed. Thus, when the order of primary and secondary diagnoses is critical $(A-B \neq B-A)$, different orders must be considered separate categories with a weight assigned to reflect their partial disagreement.

Correspondence may be addressed to Michael J Strube, Department of Psychology, Washington University, St. Louis, MO 63130.
The program also provides variance estimates under the null and non-null cases for all pairwise estimates (see Fleiss et al., 1969). This allows testing the null hypothesis that $\varkappa$ has a value of zero, or, testing $x$ against a specific nonzero alternative. Two $x$ s calculated on different samples can also be compared. A jackknife routine is also provided for the estimation of variance for multiplerater $x$ (analytic estimates are not yet available), and to provide an additional variance estimate where the large sample assumptions underlying analytic formulations (e.g., Fleiss et al., 1969) are considered untenable. The jackknife approach (Mosteller \& Tukey, 1968) is essentially a distribution-free procedure that generates $N$ estimates of $x$ by conducting the calculations with a single and different subject removed at each of the $N$ steps. Each of these $x$ estimates is then multiplied by $N-1$ and subtracted from $N$ times the $\varkappa$ based on the entire sample. The resultant values are known as pseudovalues, and their mean is the jackknife estimate of $x$. The standard error of this mean provides the jackknife estimate of variability for the $\kappa$ estimate.

Input. Data (rater responses, weights) can be entered from the keyboard or from existing sequential files. The program allows the data to be checked before proceeding with analyses. Corrected data can be saved in a sequential file for later additional analyses. The program queries the user for the location of the data and indicates the format expected. If the data do not match the expected format, the program automatically reverts to keyboard entry.

Output. The output is routed to the screen, although with program modification it can be routed to a printer. The program automatically keeps track of the number of lines printed to the screen and stops for a user keypress to advance one screen of information at a time. Because the calculation of jackknife estimates can be very timeconsuming, the computer provides an estimate of the amount of processing time required when the user is queried about the calculation of jackknife estimates.

Language. The program is written in GWBASIC (version 3.11) and is compatible with the IBM class of computers. With modification, the program can be run on other machines. The program requires $16 \mathrm{~K}$ of memory.

Availability. The program listing, which contains additional documentation, can be obtained from the author: Michael J Strube, Department of Psychology, Washington University, St. Louis, MO 63130. Alternately, a properly formatted disk and stamped return mailer can be sent to the author and a copy of the program will be returned.

\section{REFERENCES}

BLOOR, R. N. (1983). A computer program to determine interrater reliability for dichotomous-ordinal rating scales. Behavior Research Methods \& Instrumentation, 15, 615. 
Burns, E., \& Cavallaro, C. A. (1982). A computer program to determine interobserver reliability statistics. Behavior Research Methods \& Instrumentation, 14, 42.

CHAN, T. S. C. (1987). A DBASE III program that performs significance testing for the Kappa coefficient. Behavior Research Methods, Instruments, \& Computers, 19, 53-54.

CoHEN, J. (1960). A coefficient of agreement for nominal scales. Educational \& Psychological Measurement, 20, 37-46.

CoHEN, J. (1968). Weighted kappa: Nominal scale agreement with provision for scaled disagreement. Psychological Bulletin, 70, 213-220.

CONGER, A. J. (1980). Integration and generalization of kappas for multiple raters. Psychological Bulletin, 88, 322-328.

FLeiss, J. L. (1971). Measuring nominal scale agreement among many raters. Psychological Bulletin, 76, 378-382.

Fleiss, J. L., CohEN, J., \& Everitt, B. S. (1969). Large sample standard errors of kappa and weighted kappa. Psychological Bulletin, 72, 323-327.

Fleiss, J. L., Spitzer, R. L., Endicott, J., \& Cohen, J. (1972). Quan- tification of agreement in multiple psychiatric diagnoses. Archives of General Psychiatry, 26, 168-171.

Kraemer, H. C. (1980). Extension of the kappa coefficient. Biometrics, 36, 207-216.

Mosteller, F. M., \& Tukey, J. W. (1968). Data analysis, including statistics. In G. Lindzey \& E. Aronson (Eds.), Handbook of social psychology. Reading, MA: Addison-Wesley.

PowERs, S. (1985). A Pascal program that assesses the interrater reliability of nominal scales. Educational \& Psychological Measurement, 45, 613-614.

UebersaX, J. S. (1982). A generalized kappa coefficient. Educational \& Psychological Measurement, 42, 151-183.

WATKINS, M. W., \& LARIMER, L. D. (1980). Interrater agreement statistics with the microcomputer. Behavior Research Methods \& Instrumentation, 12, 466.

(Manuscript received February 10, 1989; revision accepted for publication September 8, 1989.) 